\title{
Perforation of leiomyoma of stomach
}

\author{
N.D. Merrett* and P.C. Gartell \\ Department of Surgery, Royal Hampshire County Hospital, Winchester, Hampshire, UK
}

\begin{abstract}
Summary: A case of perforation of gastric leiomyoma is reported, a presentation not previously described.
\end{abstract}

\section{Introduction}

Small clinically asymptomatic leiomyomas of the stomach are a relatively common finding at autopsy with an incidence ranging from $1 \%$ to $46 \%$. ${ }^{1.2}$ Clinically symptomatic leiomyomas are, however, relatively rare with a pre-autopsy incidence of only $1 \%$ of all gastric tumours. ${ }^{3}$ The described clinical presentations of these tumours are haematemesis and melaena, epigastric pain, epigastric mass and intraperitoneal haemorrhage. ${ }^{3-5}$ We wish to describe a case of perforation of a gastric leiomyoma of the stomach, a hitherto undescribed presentation.

\section{Clinical report}

A 46 year old woman presented to the casualty department. She had a 4-day history of intermittent epigastric pain. She had now developed a 24-hour history of left-sided lower abdominal and flank pain with no radiation. There was no history of nausea or vomiting. Bowel function and colour was normal. Past history was unremarkable. Her family history included her father having required surgery for peptic ulcer disease.

On examination, she had a temperature of $39.5^{\circ} \mathrm{C}$. She was tender in the left lower abdomen and flank with guarding. There was no rigidity or rebound tenderness. Abdominal and chest $\mathrm{X}$-rays were normal. White cell count was 10.7. Otherwise her blood count, electrolytes and serum amylase were all within normal limits. Urine microscopy showed three leucocytes and no organisms. A provisional diagnosis of diverticulitis was made and she was commenced on broad-spectrum antibiotics intravenously.

Correspondence: N. Merrett, F.R.A.C.S., 41 Riverview Avenue, Kyle Bay, NSW 2221, Australia.

*Present address: Prince Henry Hospital, Little Bay, Sydney, NSW, Australia.

Accepted: 30 October 1993
Over the next 2 days her condition improved clinically but her temperature continued to be consistently raised at $38^{\circ} \mathrm{C}$. On the third day after admission, she developed a left pleural effusion which was confirmed on chest X-ray. Ultrasonography showed a $6 \mathrm{~cm}$ left subphrenic collection. This was drained percutaneously under computed tomographic (CT) guidance and a drain left in situ. A sinogram demonstrated a subphrenic cavity communicating to the fundus of the stomach, consistent with a perforated gastric ulcer (Figure 1). As the patient was clinically well it was decided to treat her conservatively with $\mathrm{H} 2$ antagonists and nasogastric suction. A gastroscopy was not performed initially for fear that it could worsen the situation but one was performed 3 weeks after admission which showed two small ulcers in the fundus. Brush and punch biopsies were taken which demonstrated chronic inflammation on histology.

One month after admission, the drainage from the subphrenic drain had become minimal and a barium meal showed no communication to the

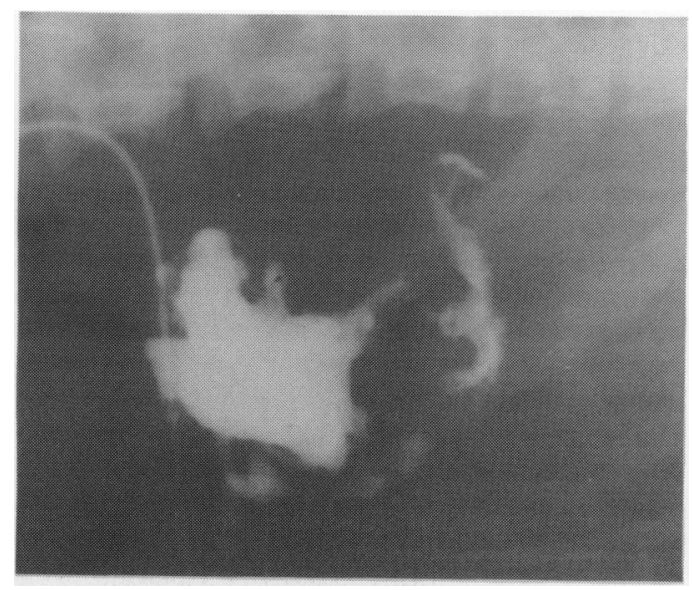

Figure 1 Sinogram of the abscess cavity showing contrast entering the stomach via an ulcer cavity. 
subphrenic cavity. The patient was discharged. A repeat gastroscopy 2 months later showed two $3 \mathrm{~mm}$ ulcers still present but now there was a strong suspicion of a submucosal mass. Histology again showed chronic inflammation. A CT scan suggested a lesion in the wall of the stomach.

At operation, the patient was found to have a $6 \mathrm{~cm}$ tumour arising from the posterior wall of stomach (Figure 2). A wedge excision of the lesion with a cuff of stomach was performed. Histological examination of the specimen showed a benign leiomyoma. The patient had an uneventful postoperative recovery.

\section{Discussion}

Gastric smooth muscle tumours usually arise in the muscularis propria and occasionally in the muscularis mucosa. They may enlarge endogastrically, intramurally or exogastrically. The majority are found in the mid- to distal stomach and are usually small, though lesions of up to $24 \mathrm{~cm}$ and $2.8 \mathrm{~kg}$ have been recorded. ${ }^{6}$

The vast majority of leiomyomas are asymptomatic. When clinically apparent, the presentation is most commonly one of epigastric pain and fullness, and bleeding. Although bleeding is evident in around $40 \%$ of clinically apparent leiomyomas, they constitute only $1 \%$ of the causes of upper gastrointestinal bleeding. Other described clinical presentations are weight loss, vomiting, early satiety and palpable mass. ${ }^{3}$

Ulceration of the overlying gastric mucosa is observed in up to $26 \%$ of clinical leiomyomas and is most commonly seen in those more than $4 \mathrm{~cm}$ in diameter. ${ }^{7}$ This ulceration is attributed to stretching and attenuation of the overlying gastric mucosa over the submucosal lesion.
Central necrosis of the tumour is also well described, this being secondary to bleeding within the tumour or growth outstripping the blood supply. ${ }^{7}$ With this high incidence of ulceration and central necrosis, it is surprising that perforation of a leiomyoma has not been described previously, although perforation of leiomyosarcomas of the stomach has been recognized.

The submucosal nature of these lesions makes endoscopic diagnosis difficult. The presence of active bleeding may also make visualization difficult. ${ }^{8}$ As they originate and enlarge beneath the mucosa, biopsy is of limited value due to the difficulty of obtaining a representative sample of tissue, with a positive yield of less than $30 \%,{ }^{5}$ and diagnosis depends on the visual recognition of a submucosal lesion, or the appearance of a submucosal lesion on a barium study. Without this recognition, treatment must be based on the presumption of a non-healing gastric ulcer, with the possibility of an underlying malignancy. More recently, endoscopic ultrasound has been described as being very useful in the diagnosis of these lesions. ${ }^{9}$

Histologically there are three recognized types of leiomyomas. ${ }^{10}$ These are ordinary leiomyomas $(66 \%)$, cellular leiomyoma ( $28 \%)$, and leiomyoma with regimentation $(8 \%) .{ }^{11}$ Leiomyoblastoma is a term also used to describe a group of tumours which macroscopically resemble leiomyomas but which display totally different histological characteristics. The major differential diagnosis of these non-epithelial tumours is that of a leiomyosarcoma, with the diagnosis hinging on the presence of metastases, bizzarre cellular changes, and the number of mitoses per high-powered field. A count of five mitoses per high-powered field is considered diagnostic of malignancy. ${ }^{12}$ This difficulty in differentiation means that a large tissue sample is

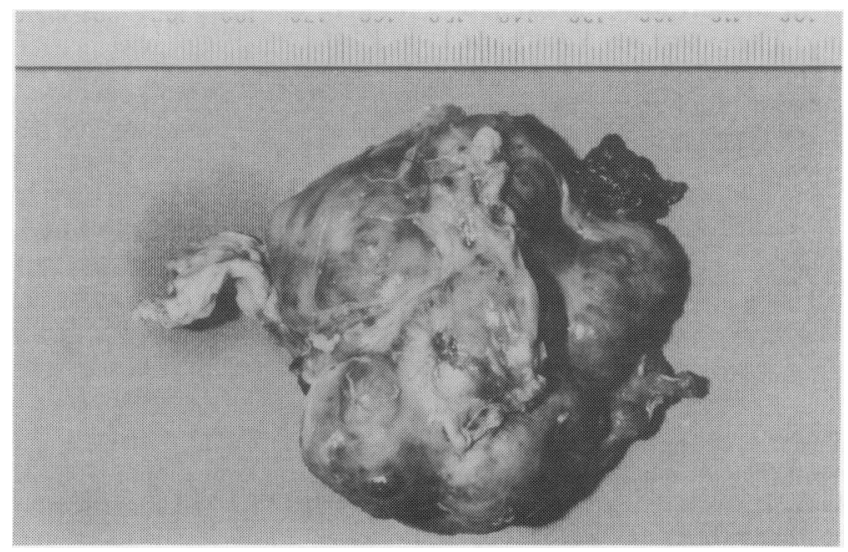

Figure 2 Photograph of serosal surface of specimen showing its size and lobulated appearance. 
required and makes frozen section and limited biopsy unreliable.

Treatment of leiomyomas and leiomyosarcomas is based on limited gastric resection. Leiomyosarcomas have a much better prognosis than adenocarcinomas as they do not exhibit local submucosal infiltration or lymphatic invasion. Their 5-year survival is described as between 30 and $60 \%$, with tumour size of $<6 \mathrm{~cm}$ and presence of metastases associated with the worse prognosis. ${ }^{6,12}$

Two salient points are illustrated by the case which we have described. Although gastric smooth

\section{References}

1. Meissner, W.A. Leiomyoma of the stomach. Arch Path 1944, 38: 207-212.

2. Kavlie, H. \& White, T.T. Leiomyomas of the upper gastrointestinal tract. Surg 1972, 71: 842-848.

3. Salmela, H. Smooth muscle tumours of the stomach. A clinical study of 112 cases. Acta Chir Scand 1968, 134: 384-391.

4. Elhamel, A., Abubaker, M. \& Wassef, S.A. Smooth muscle tumours of the stomach. It J Surg Sci 1988, 18: 233-236.

5. Halpin, R., Thomson, S.R., Catteral, N. \& Haffejee, A.A. Smooth muscle tumours of the stomach: clinicopathological aspects. J R Coll Surg Edinb 1993, 38: 23-27.

6. Cohen, S.P., Frydman, C., Zimmerman, M.J. \& Moqtaderi, F. Leiomyomatous tumours: presentation of a giant gastric leiomyoma and a review of the literature. $N$ York State J Med 1989, 89, 416-419.

7. Parry, R.G. Smooth muscle tumours of the stomach and associated gastrointestinal ulceration. Am Surg 1974, 83: $582-585$. muscle tumours are clinically unusual, they should be remembered as a possible cause of gastric ulceration and perforation. It also illustrates the difficulty in preoperative diagnosis of leiomyomas, particularly in differentiating histologically between adenocarcinomas, leiomyomas and leiomyosarcomas when there is a non-healing ulcer. Finally, at the time of operation, the surgeon must perform an excision which has an adequate margin, should the lesion subsequently prove to be a leiomyosarcoma, but not be too excessive, should it prove to be benign.

8. Senewiratne, S., Strong, R. \& Reasbeck, P.G. Smooth muscle tumours of the upper gastrointestinal tract. Austral $N$ Zealand J Surg 1987, 57: 299-302.

9. Silva, S.A., Kouzo, T., Heddeji, K. \& Kaichi, I. Endoscopic ultrasonography in leiomyoma of the gastric cardia. Surg Endosc 1989, 3: 109-111.

10. Stout, A.P. Bizarre smooth muscle tumours of the stomach. Cancer 1962, 15: 400-409.

11. Morrissey, K., Cho, E.S., Gray, G.F. \& Thorbjarnarson, B. Muscular tumours of the stomach. Clinical and pathological study of 113 cases. Ann Surg 1973, 178: 148-155.

12. Evans, H.L. Smooth muscle tumours of the gastrointestinal tract. A study of 56 cases followed for a minimum of 10 years. Cancer 1985, 56: 2242-2250. 